\title{
The Method of Fault Diagnosis for Servo System Based on Fuzzy Fault Tree Analysis
}

\author{
Fu-an Sun ${ }^{1, a}$, Cheng $\mathrm{Li}^{1, \mathrm{~b}}$, Yongming $\mathrm{Nie}^{1, \mathrm{c}}$ and Fangzhen Duan ${ }^{1, \mathrm{~d}}$ \\ ${ }^{1}$ China Satellite Maritime Tracking and Control Department, Jiangyin, Jiangsu,Chiana 214431 \\ asunfa111@163.com blicheng1860@163.com, cnwy1986@163.com, cdfz1976@163.com
}

Keywords: Servo; Fuzzy Fault Tree; Fault Diagnosis

Abstract: Aimed at the complexity of the fault diagnosis of radar servo system on the ship, the pater puts forward the method of fuzzy fault tree analysis. The paper constructs the fault tree for antenna not running. And then the quantitative analysis of the fault tree is accomplished by founding models such as exact and fuzzy fault probability of bottom things, and importance analysis of bottom things etc. Finally, the feasibility of models is validated by data calculating.

\section{Introduction}

The servo is the controlling pat of radar system, which provides a good channel for electromagnetic wave sending and receiving, by tracking spacecraft and outputting angle data. So the status of servo can determining the whether the measuring will be succeed or not. At the same time, servo is the most complex part in radar system, and contacting closely inside, which brings much difficulty for fault diagnosis of servo. Diagnosing fault by experience of persons, as a traditional method, arises many problems, such as being difficult to orientation exactly, wasting much time, and reducing life-span of equipment for checking repeatedly. So it is important to know the fault elements of servo and appraise the fault factor quantificationally.

Fault tree analysis is a method for forecasting the reliability and safety of complex system ${ }^{[1]}$, which is applied widely in many fields. The characteristic of FTA is visual, clear and reasonably, which can apply to qualitative analysis and quantitative analysis ${ }^{[2]}$. In practice, some fault factor of servo is fuzzy and hard to appraise exactly. For this, the paper appraise the fault factor with fuzzy fault tree.

\section{Establishing the Fault Tree of Representative Servo Fault}

Antenna running normally is the precondition for other function of servo. And we can detect it from soft interface, so we choose antenna running abnormally as the study object.

Mechanism Analysis for Antenna not Running. The antenna speed instruction signal is produced by antenna controlling unit (ACU). Then it transforms to current instruction signal by driver system and transfers to motor controller. At last the motor controller outputs direct current to drive electric machine to run. Further more, the transmission chain, speed-down box and electric machine are other factors for antenna running. Because the driver system works steadily and the fault probability is little, it is not analysed in the paper for convenience.

ACU computer is the main hard part of ACU. It receives and manages the tracking signal, and then outputs the speed instruction to drive antenna to track aims automatically. If the hard or soft of computer does not work well, the antenna will not run normally. The hard part of ACU computer includes main board, TTL board, AD board and DA board. The control panel of ACU, signal disposal drawer and driver interface drawer also determine whether the antenna can run normally. 
Moreover, the ACU computer connectes with other equipment through cable. For long time libration or continual inserting and pulling, the connection between cables may become bad.

Fault Tree Establishing.The fault tree analysis method regards the most obvious fault phenomena as the peak thing of fault tree, and the final cause of peak thing as the bottom thing. The middle thing reflects the causality between peak thing and bottom thing ${ }^{[3]}$. In the paper antenna running abnormally is the peak thing. According to mechanism analysis for antenna not running in last chapter, the middle thing and bottom thing can be obtained. Fig 1 is the fault tree.

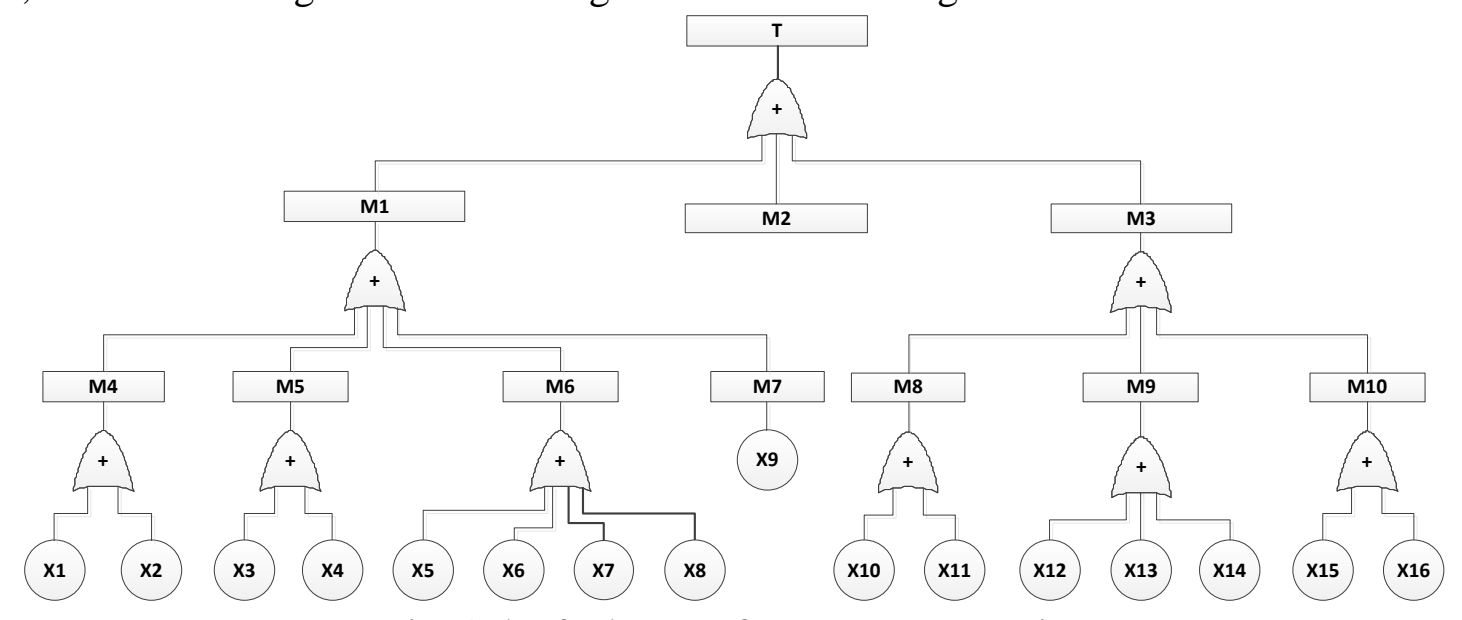

Fig. 1 the fault tree of antenna not running

In Fig. 1, T denotes antenna running abnormally, M1 denotes ACU working abnormally, M2 denotes driver system working abnormally, M3 denotes antenna structure working abnormally, M4 denotes ACU control panel working abnormally, M5 denotes signal disposal drawer working abnormally, M6 denotes ACU computer working abnormally, M7 denotes driver interface drawer working abnormally, M8 denotes transmission chain working abnormally, M9 denotes speed-down box working abnormally, M10 denotes electric machine working abnormally, X1 denotes keyswitch working abnormally, X2 denotes cable of ACU control panel working abnormally, X3 denotes power of signal disposal drawer working abnormally, X4 denotes signal disposal board working abnormally, X5 denotes TTL board, AD board and DA board working abnormally, X6 denotes cable of ACU computer working abnormally, X7 denotes soft of ACU computer working abnormally, X8 denotes mainboard of ACU computer working abnormally, X9 denotes PLC of driver interface drawer working abnormally, X10 denotes gear of transmission chain working abnormally, X11 denotes axletree of transmission chain working abnormally, X12 denotes gear and axletree of speed-down box working abnormally, X13 denotes cross-apparatus working abnormally, X14 denotes friction clutch working abnormally, X15 denotes arrester of electric machine working abnormally, X16 denotes structure of electric machine working abnormally

\section{Fuzzy Fault Tree Analysis of Representative Servo Fault}

Firstly fault tree analysis method calculates the probability of bottom thing. Secondly the probability of the peak thing is calculated on the probability of bottom thing. Lastly the importance of bottom thing is obtained. There are two kinds of probability model. One is depicted by math formula, which calculates the exact probability of bottom thing on the parameter of bottom thing. The other can not be depicted by math formula or get any parameter, which calculates the fuzzy probability by fuzzy math method.

Exact Probability Model. Aimed at the bottom thing such as X1、X2、X4、X5、X8、X9、 X10、X11、X12、X13、X14、X15andX16, the exact probability model is established. To 
establish model, the life-span distribution of bottom thing need to be known firstly. There are many distribution form, for example, Wellbull distribution, Exponential distribution and Normal distribution. Wellbull distribution is introduced in the paper.

Wellbull distribution is used in wide field, especially in the field of semiconductor. Its cumulation fault probability function and fault probability density function is ${ }^{[4]}$

$$
\begin{gathered}
F(t)= \begin{cases}1-e^{-\frac{(t-r)^{\beta}}{\alpha}} & t \geq r \\
0 & t<r\end{cases} \\
f(t)= \begin{cases}\frac{\beta}{\alpha}(t-r)^{\beta-1}-e^{-\frac{(t-r)^{\beta}}{\alpha}} & t \geq r \\
0 & t<r\end{cases}
\end{gathered}
$$

In the formula $\alpha$ is scale parameter, $\beta$ is figure parameter and $r$ is position parameter.

When $\beta<1$, the curve of $f(t)$ is monotone declining with time, and $F(t)$ declines quickly with time, which is in the early fault period. When $\beta=1, F(t)$ presents the form of constant, which is in the occasional fault period and distribution form of $f(t)$ is Exponential distribution. When $\beta>1, F(t)$ rises quickly with time, which is in the spoiled fault period. Especially when $\beta>4$, the distribution form can be regarded as Normal distribution.

$\alpha$ denotes the curve gradient of $f(t)$. The curve gradient of $f(t)$ is more steep as $\alpha$ more little.

Fuzzy Probability Model. Aimed at the bottom thing whose fault probability function is unable to establish, its fault probability is disposed with fuzzy math method, such as triangle fuzzy function, trapezia fuzzy function and normal fuzzy function. The triangle fuzzy function is introduced.

If field $U$ is real number field, $\bar{q}$ is linear triangle fuzzy number and $\mu_{\bar{q}}(x)$ is subjection function, then the subjection function is ${ }^{[5]}$

$$
\mu_{\bar{q}}(x)= \begin{cases}(x-a) /(b-a) & a \leq x<b \\ (c-x) /(c-b) & b \leq x \leq c \\ 0 & \text { 其它 }\end{cases}
$$

In the formula $x, a, b, c \in R$, and $\bar{q}=(a, b, c)$. b denotes the max subjection of $\mu_{\bar{q}}(x)$, generally speaking it is 1 . a denotes the upper limit and c denotes the lower limit. If $\bar{F}$ is the fault probability of bottom thing, then $\bar{F}=\left(F_{1}, F_{0}, F_{2}\right)$. In the formula $a=F_{1}, b=F_{0}, c=F_{2}$, which is obtained by expert marked.

For convenient calculating between exact and fuzzy probability model, the exact probability model is also disposed with fuzzy math method. Actually the parameter in the exact probability model is not absolutely certain in project, So it is reasonable. $\beta$ and $r$ is assumed to be assured and $\alpha$ is triangle fuzzy number in the paper, then the subjection function of $\bar{\alpha}$ is

$$
\mu_{\bar{\alpha}}(x)= \begin{cases}\left(x-\alpha_{1}\right) /\left(\alpha_{0}-\alpha_{1}\right) & \alpha_{1} \leq x<\alpha_{0} \\ \left(\alpha_{2}-x\right) /\left(\alpha_{2}-\alpha_{0}\right) & \alpha_{0} \leq x \leq \alpha_{2} \\ 0 & \text { other }\end{cases}
$$

According to extended principle ${ }^{[6]}$, the subjection function of $\bar{F}(t)$ is 


$$
\mu_{\bar{F}(t)}(x)= \begin{cases}\frac{\alpha_{2} \ln (1-x)+(t-r)^{\beta}}{\left(\alpha_{2}-\alpha_{0}\right) \ln (1-x)} & 1-e^{-\frac{(t-r)^{\beta}}{\alpha_{2}}} \leq x<1-e^{-\frac{(t-r)^{\beta}}{\alpha_{0}}} \\ \frac{\alpha_{1} \ln (1-x)+(t-r)^{\beta}}{\left(\alpha_{1}-\alpha_{0}\right) \ln (1-x)} & 1-e^{-\frac{(t-r)^{\beta}}{\alpha_{0}}} \leq x<1-e^{-\frac{(t-r)^{\beta}}{\alpha_{1}}} \\ 0 & \text { other }\end{cases}
$$

If $\bar{\alpha}=\left(\alpha_{1}, \alpha_{0}, \alpha_{2}\right)$, then the cumulation fault probability function is $\bar{F}(t)=\left(F_{\alpha_{2}}(t), \quad F_{\alpha_{0}}(t), F_{\alpha_{1}}(t)\right)$.

After calculating the cumulation fault probability of bottom thing, the probability is peak thing is

$$
\bar{P}(T)=\left(1-\prod_{i=1}^{16}\left[1-\bar{P}_{1}\left(X_{i}\right)\right], 1-\prod_{i=1}^{16}\left[1-\bar{P}_{0}\left(X_{i}\right)\right], 1-\prod_{i=1}^{16}\left[1-\bar{P}_{2}\left(X_{i}\right)\right]\right)
$$

Importance Analysis Model. Importance of bottom thing is the main factor for constituting the plan of fault analysis. There are many method for importance analysis such as structure importance analysis, probability importance analysis and key importance analysis. Probability importance analysis and key importance analysis are introduced in the paper.

Probability importance analysis studies the amendatory extent of the fault probability of peak thing when the state of bottom thing becomes from abnormal to normal. If the probability importance of $X_{j}$ is $I_{P}(j)$, then

$$
I_{P}\left(X_{j}\right)=\left.P(T)\right|_{P\left(X_{j}\right)=1}-\left.P(T)\right|_{P\left(X_{j}\right)=0}
$$

It can be deduced as

$$
I_{P}\left(X_{j}\right)=\frac{\partial P(T)}{\partial P\left(X_{j}\right)}=1-\left(\prod_{i=1}^{j-1}\left[1-P\left(X_{i}\right)\right] \cdot \prod_{i=j+1}^{16}\left[1-P\left(X_{i}\right)\right]\right)
$$

Key importance is the ratio between fault probability velocity of bottom thing and peak thing. If the key importance of $X_{j}$ is $I_{C}(j)$, then

$$
I_{C}\left(X_{j}\right)=\lim _{\Delta P\left(X_{j}\right) \rightarrow 0}\left[\frac{\Delta P\left(X_{j}\right)}{P\left(X_{j}\right)} / \frac{\Delta P(T)}{P(T)}\right]=\frac{P\left(X_{j}\right)}{P(T)} \frac{\partial P(T)}{\partial P\left(X_{j}\right)}=\frac{P\left(X_{j}\right)}{P(T)} I_{P}(j)
$$

According to last formula, key importance appraised the effect of bottom thing on peak thing from three aspects such as fault probability of bottom thing, fault probability of peak thing and probability importance of bottom thing. So key importance appraises the effect of bottom thing on peak thing more reasonably. In the process of fault analysis and daily maintenance, key importance can be a gist for attention to bottom thing .

Case Analysis. Hypothetically the servo system is in the occasional fault period, then the distribution form of $f(t)$ become as Normal distribution and $\beta=1$. If $r=0$, then

$$
F(t)= \begin{cases}1-e^{-\frac{t}{\alpha}} & t \geq 0 \\ 0 & t<0\end{cases}
$$

In the formula $\alpha$ is mean time between failures (MTBF) of bottom thing actually. For some reason the exact parameter of servo equipment is unable to be presented. The rude MTBF of X1, X3, $\mathrm{X} 4, \mathrm{X} 5, \mathrm{X} 8, \mathrm{X} 9, \mathrm{X} 10, \mathrm{X} 11, \mathrm{X} 12, \mathrm{X} 13, \mathrm{X} 14, \mathrm{X} 15$ and X16 is presented and disposed fuzzy. For example, $\bar{\alpha}_{\mathrm{X} 1}=(4800,5000,5200), \bar{\alpha}_{\mathrm{X} 3}=(1900,2000,2100), \bar{\alpha}_{\mathrm{X} 4}=(1700,1800,1900)$, $\bar{\alpha}_{\mathrm{X} 5}=(1900,2000,2100), \quad \bar{\alpha}_{\mathrm{X} 8}=(2400,2500,2600), \quad \bar{\alpha}_{\mathrm{X} 9}=(3800,4000,4200)$, $\bar{\alpha}_{\mathrm{X} 10}=(8600,9000,9400), \quad \bar{\alpha}_{\mathrm{x} 11}=(8600,9000,9400), \bar{\alpha}_{\mathrm{X} 12}=(9600,10000,10400)$, 
$\bar{\alpha}_{\mathrm{x} 13}=(7700,8000,8300), \quad \bar{\alpha}_{\mathrm{X} 14}=(6700,7000,7300), \quad \bar{\alpha}_{\mathrm{x} 15}=(8600,9000,9400)$ and

$\bar{\alpha}_{\mathrm{X} 16}=(9600,10000,10400)$.

If $\mathrm{t}=500$, then $\bar{P}\left(X_{1}\right)=(0.0989,0.0952,0.0917), \quad \bar{P}\left(X_{3}\right)=(0.2314,0.2212,0.2119)$,

$\bar{P}\left(X_{4}\right)=(0.1233,0.1175,0.1122), \quad \bar{P}\left(X_{5}\right)=(0.2314,0.2212,0.2119)$,

$\bar{P}\left(X_{8}\right)=(0.1881,0.1813,0.1749), \quad \bar{P}\left(X_{9}\right)=(0.1233,0.1175,0.1122)$,

$\bar{P}\left(X_{10}\right)=(0.0565,0.0540,0.0518), \quad \bar{P}\left(X_{11}\right)=(0.0565,0.0540,0.0518)$,

$\bar{P}\left(X_{12}\right)=(0.0508,0.0488,0.0469), \quad \bar{P}\left(X_{13}\right)=(0.0629,0.0606,0.0585)$,

$\bar{P}\left(X_{14}\right)=(0.0719,0.0689,0.0662), \quad \bar{P}\left(X_{15}\right)=(0.0565,0.0540,0.0518)$ and

$\bar{P}\left(X_{16}\right)=(0.0508,0.0488,0.0469)$.

According to the expert marking and statistical data, the fuzzy probability of X2、X6、X7 can be obtained. For example $\bar{P}\left(X_{2}\right)=\left(\begin{array}{ll}0.1233, & 0.1175,0.1122), \quad \bar{P}\left(X_{6}\right)=(0.1233,0.1175,0.1122)\end{array}\right.$ and $\bar{P}\left(X_{7}\right)=(0.1635,0.1535,0.1447)$.

Based on the key importance formula, key importance of every bottom thing can be obtained. The max subjection key importance is presented such as $I_{C}\left(X_{1}\right)=0.0936, I_{C}\left(X_{2}\right)=0.1139$,

$I_{C}\left(X_{3}\right)=0.2093, \quad I_{C}\left(X_{4}\right)=0.2282, \quad I_{C}\left(X_{5}\right)=0.2093, \quad I_{C}\left(X_{6}\right)=0.1139, \quad I_{C}\left(X_{7}\right)=0.1477$,

$I_{C}\left(X_{8}\right)=0.1733, \quad I_{C}\left(X_{9}\right)=0.1139, \quad I_{C}\left(X_{10}\right)=0.0530, \quad I_{C}\left(X_{11}\right)=0.0530, \quad I_{C}\left(X_{12}\right)=0.0479$,

$I_{C}\left(X_{13}\right)=0.0593, I_{C}\left(X_{14}\right)=0.0674, I_{C}\left(X_{15}\right)=0.0530$ and $I_{C}\left(X_{16}\right)=0.0479$ 。

$I_{C}\left(X_{4}\right)>I_{C}\left(X_{3}\right)=I_{C}\left(X_{5}\right)>I_{C}\left(X_{8}\right)>I_{C}\left(X_{7}\right)>I_{C}\left(X_{2}\right)=I_{C}\left(X_{6}\right)=I_{C}\left(X_{9}\right)>I_{C}\left(X_{1}\right)>I_{C}\left(X_{14}\right)>$ $I_{C}\left(X_{13}\right)=I_{C}\left(X_{10}\right)=I_{C}\left(X_{11}\right)=I_{C}\left(X_{15}\right)>I_{C}\left(X_{12}\right)>I_{C}\left(X_{16}\right)$

According to the taxis of key importance, the signal disposal board should be checked firstly when antenna runs abnormally.

\section{Conclusion}

The mechanism analysis of antenna not running is expressed directly by fault tree. The importance model of bottom thing is established with fuzzy math method. The model can be used to quantitatively analysis on fault diagnosis. At last the feasibility of model is validated by a case. However the quantitatively analysis of fault tree only thinks about the fault probability of bottom thing. Next step integrative appraising index system will be established in order to study the importance of bottom thing on peak thing more roundly, which is a base for the expert system of servo fault diagnosis.

\section{References}

[1] Li Xin. A New Study of Fuzzy Fault Tree Analysis[J]. Electronic Product Reliability and Environment Test, 2007(1): 27-30

[2] Cui Ming. Study on Security of Electric Bicycle Based on Fault Tree[J]. Journal of inner Mognolia University (Natural Science Edition), 2011(4): 467-471

[3] Zheng Leilei, Song Lihua, Guo Rui etc. Application of FAT in Information Security Risk Assessment[J]. Computer Science, 2011(10):106-108

[4] Lu Qiqing, Zhang Ankang. Dependability and Inefficiency Analysis of Semiconductor [M]. Jiangsu science and Technology Press, 1981. 
[5] Hu Shengwu. Quality Assessment and Dependability Analysis of GIS[M]. Beijing:Sino Maps Press, 2006.

[6] Wang Haitao, Wang Jingqin, Nie Junlan. Reliability Study of Low Voltage Switchgear based on Trapezoidal Fuzzy Number[J]. Low Voltage Apparatus, 2011(18):9-11 